\title{
Operationalizing Treat-to-Target for Osteoporosis
}

\author{
E. Michael Lewiecki \\ New Mexico Clinical Research \& Osteoporosis Center, Albuquerque, NM, USA
}

Treat-to-target (TTT) for osteoporosis is a concept for individualizing patient treatment decisions that focuses on achieving an acceptable level of fracture risk rather than response to treatment alone. While a response to treatment is essential in order to achieve an acceptable level of risk, it is not necessarily sufficient. Some patients have a good response to treatment yet remain at high level of fracture risk. Since there is no way to directly measure bone strength in patients treated for osteoporosis, a surrogate measurement must be used. Bone mineral density (BMD) is commonly used to select patients for treatment and has emerged as the most useful surrogate for assessing reduction of fracture risk after treatment is started. Recent large meta-regression studies have shown a robust correlation between larger increases in BMD with treatment and greater reductions in fracture risk. Application of TTT for osteoporosis involves assessing fracture risk before starting treatment and initiating treatment with an agent that is most likely to reduce fracture risk to an acceptable level, represented by a target BMD T-score, over a reasonable period of time. This review offers suggestions for implementing TTT for osteoporosis in clinical practice and managing patients who fail or succeed in reaching the target. More study is needed to fully validate the use of TTT for osteoporosis for initiating and modifying treatments to reduce fracture risk.

Keywords: Osteoporosis; Therapeutics; Bone and bones; Fractures, bone; Goals; Target

\section{INTRODUCTION}

Treat-to-target (TTT; sometimes called treat-to-goal or goal-directed treatment) is the concept that treatment decisions should be made according to the likelihood of achieving a measure or composite of measures that represents treatment success. These biomarkers are surrogates for the clinical outcome of interest, which may be the absence of an adverse event with a silent chronic disease or disease remission with a chronic symptomatic disease. This strategy of disease management is familiar to most physicians for its application to the care of patients with conditions such as hypertension [1], diabetes mellitus [2], and rheumatoid arthritis [3].

Osteoporosis is a silent chronic disease characterized by reduced bone strength and increased risk of fracture [4]. Fracture

Received: 20 January 2021, Revised: 3 February 2021,

Accepted: 7 February 2021

Corresponding author: E. Michael Lewiecki

New Mexico Clinical Research \& Osteoporosis Center, 300 Oak St. NE, Albuquerque, NM 87106, USA

Tel: +1-505-855-5525, Fax: +1-505-884-4006, E-mail: mlewiecki@gmail.com is the clinical outcome of interest for patients with osteoporosis; the goal of treatment is to prevent fractures. Since the risk of fracture can never be totally eliminated, the pragmatic treatment goal is reduction of fracture risk. This raises several questions regarding treatment decisions.

1. How much reduction in fracture risk is desirable and achievable?

2. How can we best assess the reduction in fracture risk with treatment?

3. How can we use this information to individualize treatment decisions?

All medications approved for the treatment of osteoporosis have been shown to reduce the risk of vertebral fractures in randomized controlled clinical trials (RCTs) conducted in postmenopausal women with osteoporosis. For appropriately select-

Copyright $(\odot 2021$ Korean Endocrine Society

This is an Open Access article distributed under the terms of the Creative Commons Attribution Non-Commercial License (https://creativecommons.org/ licenses/by-nc/4.0/) which permits unrestricted non-commercial use, distribution, and reproduction in any medium, provided the original work is properly cited. 
ed patients, any treatment is probably better than none; however, not all drugs are the same.

Bisphosphonates (e.g., alendronate, risedronate, ibandronate, zoledronate) constitute the most widely used class of drugs for the treatment of osteoporosis because of low cost and a generally favorable balance of benefits and risks. These antiresorptive agents are associated with modest increases in bone mineral density (BMD) and reductions in fracture risk [5]. Since the introduction of bisphosphonates for the treatment of osteoporosis, other therapeutic agents with differing pharmacological and clinical profiles have been developed. These include a more potent antiresorptive compound, denosumab, that increases BMD more than bisphosphonates [6-8], and anabolic compounds that reduce fracture risk more than bisphosphonates [9-12], with some anabolic compounds increasing BMD more than others $[13,14]$. These new developments, along with robust data showing that larger increases of BMD with treatment are associated with greater reductions of fracture risk $[15,16]$ and recognition of the importance of treatment sequence [17] have increased the complexity of choosing initial osteoporosis therapy and assessing the outcomes of treatment.

In 2013, the idea of facilitating the management of patients with osteoporosis using a TTT strategy was presented in two simultaneous publications $[18,19]$. The principles supporting the concept of TTT for osteoporosis were described (Fig. 1) and recommendations for further action were made. These publications raised awareness of this concept in the medical community and quickly generated lively discussions on the merits and limitations of TTT for osteoporosis [20,21]. This is an update of progress with TTT for osteoporosis, including new data that have emerged.

\section{AMERICAN SOCIETY FOR BONE AND MINERAL RESEARCH: NATIONAL OSTEOPOROSIS FOUNDATION WORKING GROUP PROGRESS REPORT (2017)}

Following the two initial publications on TTT for osteoporosis, the American Society for Bone and Mineral Research (AS-
BMR) joined with the US National Osteoporosis Foundation (NOF) to establish a working group, composed of leading osteoporosis experts and clinicians from seven different countries, representing a range of medical specialties, to review the best available medical evidence, identify gaps in the evidence, and propose a pathway forward. The Working Group published a progress report in 2017 [22]. Recent evidence is currently under review in preparation for a final report.

The ASBMR-NOF Working Group described differences between standard treatment (sometimes called "step therapy" or "fail first") and the TTT approach (Fig. 2) [22,23]. It was proposed that treatment targets be predicated on the initial indication for treatment. When a patient is started on treatment because of T-score $\leq-2.5$, then the target would be a T-score that is at least above the T-score treatment threshold of -2.5, ideally much better. When a patient is started on treatment because of 10 -year fracture probability by a fracture risk algorithm, such as FRAX, that is above the country-specific treatment threshold (e.g., major osteoporotic fracture risk $\geq 20 \%$ or hip fracture risk $\geq 3 \%$ in the United States), the target would be a level of risk that is at least above the fracture risk treatment threshold, ideally much better. When a patient is treated because of having an osteoporotic fracture despite having T-score $>-2.5$, the target is no future fracture, as it is with all patients. Although the TTT approach is attractive for physicians due to its intuitive nature, it has been more aspirational than achievable in some respects (e.g., there was, and still is, no validated fracture risk algorithm that captures the reduction in fracture risk associated with treatment) and could not have been applied to all patients treated for osteoporosis. More evidence was needed before TTT for osteoporosis could be accepted for general use in clinical practice.

To fully validate the use of TTT for osteoporosis, a research agenda was proposed by the Working Group and by others [20]. This included studies to identify a level of T-score or fracture risk at which anabolic therapy should be considered; to better define the correlation between BMD increases with treatment and fracture risk reduction; to compare the probability of reaching a T-score target with different therapeutic agents; head-tohead trials comparing the efficacy of therapeutic agents to reduce

- Initial treatment should be selected according to the likelihood of achieving an acceptable level of fracture risk over a reasonable period of time.

- A patient may respond to therapy yet still have an unacceptably high risk of fracture.

- Response to therapy is essential but not necessarily sufficient in achieving an acceptable level of risk.

Fig. 1. Principles of treat-to-target for osteoporosis [18,19]. Application of these principles provides a foundation for individualizing treatment decisions according to the baseline level of fracture risk and desired magnitude of risk reduction. 
Standard treatment: Treatment success is defined as response to therapy and no incident fracture. Response is usually defined as stability or an increase in bone mineral density and/or an appropriate change of a bone turnover marker.

- Step 1. Begin treatment with a low-cost generic oral antiresorptive (e.g., alendronate), provided there is no contraindication

- Step 2. If the patient fails Step 1 due to a contraindication, adverse effect, or poor response, switch to another low-cost antiresorptive (e.g., zoledronic acid)

- Step 3. If the patient fails Step 2, consider more potent antiresorptive (e.g., denosumab) or anabolic therapy

Treat-to-target: Treatment success is defined as achievement of a target level of a biomarker (e.g., a specified T-score), representing an acceptable level of fracture risk, and no incident fracture.

- Treatment target is established as part of the baseline evaluation.

- Initial treatment is selected based on fracture risk stratification and the probability of reaching the treatment target over a reasonable period of time, with consideration of more aggressive therapy for patients at higher risk of fracture.

- Progress toward reaching the target is monitored periodically, with decisions to stop, continue, or change therapy based on progress toward or achievement of the target.

Fig. 2. Standard treatment vs. treat-to-target for osteoporosis [22,23]. Standard treatment ("step therapy") is often preferred by public health officials and payors of healthcare services due to low initial treatment costs. Treat-to-target may have higher initial costs but individualizes treatment decisions, potentially offering a greater chance of treatment success.

fracture risk; and assessment of benefits with switching from one anti-fracture medication to another. The ultimate test for the clinical utility of TTT for osteoporosis would be a study comparing anti-fracture efficacy using TTT versus standard care.

In recent years, much but not all of the proposed research agenda has been addressed. Two new anabolic compounds (abaloparatide, romosozumab) for treating osteoporosis have become available. Head-to-head clinical trials with fractures as endpoints have been conducted. The importance of treatment sequence is now better appreciated. Clinical practice guidelines have been revised to incorporate new methods for fracture risk stratification as a guide for selecting initial therapy. TTT for the United States has been included in the latest 'Clinician's Guide to Prevention and Treatment of Osteoporosis' by the NOF [24] and its use supported by several European consensus statements $[25,26]$. Although there is no cure for osteoporosis, treatment outcomes that were once thought aspirational are now, at least in part, achievable. More is yet to be done, but important progress has been made in addressing knowledge gaps regarding TTT for osteoporosis and our understanding of how to apply TTT to the management of patients with osteoporosis.

\section{MEASURING TREATMENT RESPONSE AND NON-RESPONSE}

Prior to the approval of denosumab in 2010, treatment options were largely limited to bisphosphonates, with fewer patients being treated with teriparatide. The expected BMD increases with bisphosphonates were small compared with what can now be achieved with newer treatment regimens. The pattern of BMD change was typically an initial increase over several years followed by a plateau that persisted with continuing therapy. With bisphosphonates, an acceptable response to treatment was considered to be an increase or stability of BMD, in which case continuing treatment was usually recommended. A poor response to treatment was described as a significant decrease in BMD, lack of expected change in a bone turnover marker, or possibly a fracture on therapy, any of which might trigger investigation for underlying causes and possibly a change in therapy [23]. In 2012, a working group of the International Osteoporosis Foundation (IOF) identified three circumstances of "treatment failure," largely based on expert opinion, that warranted a treatment change: (1) two or more incident fractures during treatment; (2) one incident fracture plus lack of suppression of bone turnover markers with antiresorptive therapy and/or significant decrease of BMD; or (3) lack of suppression of bone turnover markers and significant decrease of BMD [27]. For such patients, it was suggested that a weaker antiresorptive agent be replaced by one that is more potent, an oral drug be replaced by an injected drug, and a potent antiresorptive drug be replaced by an anabolic drug. These considerations may also be applicable to a patient who is responding to therapy yet remains at high risk for fracture.

\section{MEASURING TREATMENT SUCCESS}

Response to treatment is necessary and desirable in order to reduce fracture risk. However, with the emergence of new drugs 
and new data on comparative efficacy, it became apparent that some drugs or sequences of treatment could increase BMD and reduce fracture risk more than others. This in turn led to consideration of biomarkers that could be used in clinical practice as surrogates for fracture risk reduction with treatment. Three candidate biomarkers came to the forefront: BMD, bone turnover markers, and a fracture risk algorithm (yet to be created) for treated patients. BMD, expressed as T-score (the standard deviation difference between the patient's BMD and the mean BMD of a young-adult reference population), has emerged as the most useful marker of treatment success with TTT for osteoporosis, with robust supporting data.

In an analysis of 13 RCTs with antiresorptive agents by Wasnich and Miller [28], published in 2000, it was found that larger increases in BMD with treatment were associated with greater reductions in vertebral fracture risk. The poison regression model showed that an $8 \%$ increase in lumbar spine BMD would reduce vertebral fracture risk by $54 \%$; a $5 \%$ increase in hip BMD was associated with a $50 \%$ decrease of vertebral fracture risk. There was a small but significant decrease of fracture risk with no measurable increase in BMD, probably attributable to a modest decrease of bone remodeling that is not measured well with BMD by dual-energy X-ray absorptiometry (DXA). This study was soon followed by other similar analyses of published RCTs showing that larger increases in lumbar spine BMD accounted for greater reduction in vertebral fracture risk [29] and that larger increases in lumbar spine or hip BMD accounted for greater reduction of non-vertebral fracture risk [30]. Using different methodologies, these studies found differences in the magnitude of fracture risk reduction due to BMD as opposed to other factors (e.g., decrease of bone remodeling); however, they were consistent in showing that fracture risk reduction was proportional to BMD increase.

The largest study to date to evaluate the contribution of BMD change with treatment to fracture risk reduction is the Foundation for the National Institutes of Health (FNIH) Bone Quality Study by Bouxsein et al. [15]. This was a meta-regression of published data from 28 RCTs involving 19 different therapeutic agents (six bisphosphonates, four selective estrogen receptor modulators, calcitonin, estrogen, tibolone, calcitonin, denosumab, two parathyroid hormone analogs, romosozumab, and odanacatib). Trial size ranged from 246 to over 16,000 subjects with a total of over 100,000 subjects and study durations that ranged from 1 to 8 years. Analyses of this remarkably robust dataset confirmed and extended the findings of earlier studies, showing that larger improvements of BMD measured by DXA were as- sociated with greater reductions in fracture risk. The association was strong for BMD at the total hip, femoral neck, and lumbar spine with vertebral fractures, and for BMD at the total hip and femoral neck with hip fractures, with a weak association (not statistically significant) between BMD at the total hip, femoral neck, and lumbar spine with non-vertebral fractures.

There are limitations with the use of published trial data in the FNIH meta-regression, such as inconsistency of study durations and fracture definitions. To address these limitations and further evaluate the association between BMD increase with treatment and fracture risk reduction, the FNIH Bone Quality Project conducted another meta-regression that used a unique dataset of individual patient data [16]. In this analysis, individual patient data from 91,779 participants of 23 RCTs were analyzed. Significant associations were found between treatment-related changes in total hip, femoral neck, and lumbar spine BMD and reductions in vertebral $(P=0.0005)$, hip $(P=0.023)$, and nonvertebral fractures $(P<0.0001)$. Hip BMD changes explained $44 \%$ to $67 \%$ of treatment-related fracture risk reduction. The two FNIH studies provide strong evidence that increases in BMD with treatment may be a useful surrogate endpoint for fractures in the design of clinical trials for new therapeutic agents. This also supports the concept that it is not necessary that BMD increase with treatment explains the entirety of fracture risk reduction in order for BMD to be a useful clinical endpoint; it is enough that the association is proportional. While the results do not necessarily apply to the care of individual patients, the findings are consistent with the use of BMD as a treatment target in clinical practice.

Since the concept of using BMD T-score as a treatment target for osteoporosis was first introduced $[18,19]$ and initial skepticism regarding its clinical utility was expressed [20], support for the use of TTT for osteoporosis has come forth from a range of sources [25,26,31]. However, there has been lack of consensus on what the target T-score should be, how long to reach to target, which skeletal site should be measured, what level of fracture risk with treatment is acceptable, and whether treatment should be different for different patients and different drugs. There is evidence from the Fracture Reduction Evaluation of Denosumab in Osteoporosis Every 6 Months (FREEDOM) trial and its longterm extension (up to 10 years of continuous treatment with denosumab) that the incidence of nonvertebral fractures is lower with higher total hip T-score, with a plateau of fracture incidence with achievement of total hip T-score between -2.0 and -1.5 [32]. This correlation was independent across important demographic variables, including age and prior fractures. It is also no- 
table that for women with baseline T-scores between -2.1 and -2.5 , a 1.0 T-score unit increase with denosumab was associated with a significant decrease of nonvertebral fracture risk. Taken as a whole, these data support the use of total hip T-score target of at least -2.0 and perhaps higher, at least for treatment with denosumab. Although it is not known with certainty that the same T-score targets would apply to treatment with other pharmacological agents, it is biologically plausible and supported by data from the FNIH meta-regression [16].

\section{SELECTING INITIAL TREATMENT}

Clinical practice guidelines for osteoporosis have traditionally provided indications for treatment based on fracture risk, BMD, and/or prior fracture, but offered little guidance on which therapeutic agent should be selected for initiation of treatment. This has changed with recent guidelines [33-35] that suggest consideration of different treatment options depending on the level of fracture risk. In general, it is recommended that low risk patients be managed with non-pharmacological therapy. Patients at high risk could be treated with any one of many therapeutic options, including bisphosphonates, denosumab, and raloxifene. A new category of "very high risk" has been identified to distinguish patients who are candidates for consideration of initiating treatment with an anabolic agent such as teriparatide, abaloparatide, or romosozumab.

\section{APPLICATIONS OF TTT FOR OSTEOPOROSIS IN CLINICAL PRACTICE}

The concept of TTT for osteoporosis provides an approach for managing patients with osteoporosis that may coincide with the conventional approach of starting treatment with an oral bisphosphonate for most patients, but may lead to other treatment choices for some patients. Designation of a target T-score does not necessarily mean that it is achievable, just as treatment targets for other disorders are not always achievable, but nevertheless being on a pathway toward the target may be beneficial. Fig. 3 describes some pragmatic ways in which TTT for osteoporosis might be used in clinical practice [27,33-42]. Below are

\footnotetext{
- Evaluation before starting treatment: All patients should be evaluated for factors contributing to skeletal fragility and fracture risk [37], with appropriate interventions for those that are correctable. Fracture risk should be assessed considering all available clinical information, including bone mineral density (BMD), clinical risk factors for fracture, and spine imaging, when appropriate, for identification of vertebral fractures [38]. Imaging of the spine can be conveniently done with vertebral fracture assessment (VFA) by dual-energy X-ray absorptiometry (DXA) at the same time BMD is measured, or by conventional radiography.

- Identifying a treatment target: When treatment is started because of T-score $\leq-2.5$, a reasonable treatment target is T-score at least $>-2.0$. When treatment is started with T-score $>-2.5$, consider a target of increasing BMD by at least $1.0 \mathrm{~T}$-score units.

- Selecting initial treatment: Treatment should be started with a medication that is likely to reach the treatment target in a reasonable period of time (e.g., 2 to 3 years), with greater urgency for patients at very high risk for fracture, especially those with a recent major fracture. Current clinical practice guidelines from endocrinology organizations have suggestions for selecting initial therapy based on level of fracture risk, with anabolic therapy a consideration as initial therapy when fracture risk is very high [33-35].

- Monitoring treatment: Patients should be monitored for treatment efficacy and safety, as well as to assess progress toward the treatment target. This is typically accomplished by measuring BMD every 1 to 2 years according to clinical circumstances and availability of DXA. Repeating spine imaging may be helpful for identifying incident vertebral fractures [39], recognition of which might lead to a change in therapy.

- When the patient is not on a pathway to reach the treatment target or there is a change in fracture risk assessment: When a patient is not responding to treatment, or responding to treatment but not likely to reach the treatment target, or has a change in fracture risk according to having a new fracture, consideration should be given to a change in therapy. This may be switching from a mild antiresorptive drug to one that is more potent, or switching from an antiresorptive drug to an anabolic drug, or possibly adding an anabolic to an antiresorptive [27].

- When the patient is on a pathway to reaching the treatment target: Continue treatment.

-When the treatment target is reached: Since osteoporosis is a lifelong disease, lifelong attention to reducing fracture risk is warranted [40]. Patients who have reached a treatment target after completing a course of therapy with an anabolic agent should be switched to an antiresorptive drug to enhance and consolidate the benefits achieved. Patients who have reached a treatment target with long-term bisphosphonate therapy are potential candidates for a bisphosphonate holiday [41], with appropriate monitoring and resumption of treatment when the therapeutic effect has dissipated. For those who have reached a treatment target with denosumab, the same treatment could be continued or the patient switched to a bisphosphonate [36]. The risk of osteonecrosis of the jaw with long-term therapy may be minimized by maintaining good oral hygiene. Long femur imaging by DXA may be helpful to recognize focal cortical thickening that is associated with atypical femur fractures [42].
}

Fig. 3. Integrating treat-to-target for osteoporosis into clinical practice. These are suggestions for using the treat-to-target concepts to individualize treatment decisions for patients with osteoporosis. 
a few examples of clinical scenarios where TTT might influence treatment.

\section{Treatment indication: T-score $\leq \mathbf{- 2 . 5}$}

A 77-year-old woman is frail and has fallen three times in the past year. She has no known fracture and has never received pharmacological therapy to reduce fracture risk. She is a cigarette smoker (1 pack per day since age 18 years). Her mother had a hip fracture from a fall at age 73 years. The DXA study shows left total hip T-score -3.1 . What treatment should be started?

This patient has very low BMD and multiple risk factors for fracture. Starting treatment with alendronate might be expected to increase total hip BMD by about $3.4 \%$ over 4 years [43], perhaps increasing her T-score to -2.8 or -2.7 followed by a plateau of BMD at this level with continuing the same treatment. This would represent a good response to therapy but fracture risk would still be high. The 2020 clinical practice guidelines of the American Association of Clinical Endocrinologists/American College of Endocrinologists (AACE/ACE) classify her as being at very high fracture risk due to T-score $<-3.0$ and suggest that she is a candidate for consideration of anabolic therapy.

In the Active-Controlled Fracture Study in Postmenopausal Women with Osteoporosis at High Risk (ARCH), treatment with romosozumab was superior to alendronate for reducing the risk of new radiographic vertebral fractures and clinical fractures in postmenopausal women at very high risk for fracture [9]. In the Fracture Study in Postmenopausal Women with Osteoporosis (FRAME), 1 year of romosozumab followed by 2 years of denosumab is associated with a total hip BMD increase of $8.8 \%$ over 3 years, more than twice the expected BMD increase with alendronate.

For this patient, treatment with romosozumab for 1 year followed by denosumab could increase total hip T-score to -2.3 or -2.2 over 3 years; with continued use of denosumab, it is likely that she could reach a T-score target of $>-2.0$, with optimization of fracture risk reduction [32]. Once the treatment target is achieved, denosumab could be continued, recognizing that no clinical trials have evaluated efficacy or safety beyond 10 years, or consideration could be given to switching to a bisphosphonate, although the ideal way to do so is uncertain [36].

Treatment indication: high fracture risk with T-score $>\mathbf{- 2 . 5}$ Pharmacological therapy to reduce fracture risk may be indicated for patients with a previous fracture, especially a recent one, or when a fracture risk algorithm, such as FRAX, shows a level of risk that exceeds country-specific intervention thresholds, even with T-scores $>-2.5$. In such patients, an increase of at least 1.0 T-score units may be a pragmatic treatment target, as suggested with patients having baseline T-score between -2.1 and -2.5 treated with denosumab [32].

\section{STRENGTHS AND LIMITATIONS}

The robust correlation between BMD increases with treatment and fracture risk reduction, demonstrated in the FNIH studies and others, suggests that BMD could be used as a surrogate for fracture risk in clinical trials of new therapeutic agents for osteoporosis and provides support for utilizing BMD as a treatment target in clinical practice. Head-to-head trials showing that BMD increases more with some drugs than others, and that some drugs reduce fracture risk more than others, highlights the importance of matching treatment choices to the patient's level of risk. Recent appreciation that the sequence of therapy makes a difference in therapeutic response is cause to take care in selection of the drug or drug class for initiating treatment. A Tscore target of $>-2.0$, and perhaps better yet -1.5 , is supported by long-term data with denosumab.

More data are needed to assess the clinical utility of a T-score target with all approved drugs and a range of patient demographics. Consensus needs to be reached on using a uniform T-score target for all drugs, a different T-score target for different drugs, or other targets depending on clinical circumstances. Consensus is needed on what constitutes an acceptable level of fracture risk and whether that might be different for different patients. Data on the probability of reaching a defined T-score over a specified period of time with different therapeutic agents is needed, along with data on changes of fracture risk associated with switching from one therapeutic agent to another. TTT must be widely incorporated into treatment guidelines worldwide before it will be fully accepted and implemented in clinical practice.

\section{CONCLUSIONS}

TTT for osteoporosis is a concept that provides guidance for selection of initial treatment and changing treatment based on the likelihood of achieving and maintaining an acceptable level of fracture risk. It provides clinicians with a structure for individualizing treatment decisions and optimizing outcomes.

\section{CONFLICTS OF INTEREST}

The author has received no direct income from potentially con- 
flicting entities. His employer, New Mexico Clinical Research $\&$ Osteoporosis Center, has received research grants from Radius, Amgen, Mereo, Bindex; income for service on scientific advisory boards or consulting for Amgen, Radius; service on speakers' bureaus for Radius, Alexion; project development for University of New Mexico; and royalties from UpToDate for sections on DXA, fracture risk assessment, and prevention of osteoporosis. He is a board member of the National Osteoporosis Foundation and Osteoporosis Foundation of New Mexico. He has served on guideline committees for the International Society for Clinical Densitometry, National Osteoporosis Foundation, American Association of Clinical Endocrinologists, and North American Menopause Society.

The author has received no external financial support and no assistance in the conceptualization, writing, and editing of this manuscript.

\section{ORCID}

E. Michael Lewiecki https://orcid.org/0000-0003-2026-9587

\section{REFERENCES}

1. Verdecchia P, Reboldi G, Angeli F. The 2020 International Society of Hypertension global hypertension practice guidelines: key messages and clinical considerations. Eur J Intern Med 2020;82:1-6.

2. American Diabetes Association. 6. Glycemic targets: standards of medical care in diabetes-2020. Diabetes Care 2020;43(Suppl 1):S66-76.

3. Smolen JS, Landewe RBM, Bijlsma JWJ, Burmester GR, Dougados M, Kerschbaumer A, et al. EULAR recommendations for the management of rheumatoid arthritis with synthetic and biological disease-modifying antirheumatic drugs: 2019 update. Ann Rheum Dis 2020;79:685-99.

4. NIH Consensus Development Panel on Osteoporosis Prevention, Diagnosis, and Therapy. Osteoporosis prevention, diagnosis, and therapy. JAMA 2001;285:785-95.

5. Khosla S, Bilezikian JP, Dempster DW, Lewiecki EM, Miller PD, Neer RM, et al. Benefits and risks of bisphosphonate therapy for osteoporosis. J Clin Endocrinol Metab 2012;97: 2272-82.

6. Brown JP, Prince RL, Deal C, Recker RR, Kiel DP, de Gregorio LH, et al. Comparison of the effect of denosumab and alendronate on BMD and biochemical markers of bone turnover in postmenopausal women with low bone mass: a ran- domized, blinded, phase 3 trial. J Bone Miner Res 2009;24: 153-61.

7. Brown JP, Roux C, Ho PR, Bolognese MA, Hall J, Bone $\mathrm{HG}$, et al. Denosumab significantly increases bone mineral density and reduces bone turnover compared with monthly oral ibandronate and risedronate in postmenopausal women who remained at higher risk for fracture despite previous suboptimal treatment with an oral bisphosphonate. Osteoporos Int 2014;25:1953-61.

8. Miller PD, Pannacciulli N, Brown JP, Czerwinski E, Nedergaard BS, Bolognese MA, et al. Denosumab or zoledronic acid in postmenopausal women with osteoporosis previously treated with oral bisphosphonates. J Clin Endocrinol Metab 2016;101:3163-70.

9. Saag KG, Petersen J, Brandi ML, Karaplis AC, Lorentzon $\mathrm{M}$, Thomas T, et al. Romosozumab or alendronate for fracture prevention in women with osteoporosis. N Engl J Med 2017;377:1417-27.

10. Saag KG, Shane E, Boonen S, Marin F, Donley DW, Taylor $\mathrm{KA}$, et al. Teriparatide or alendronate in glucocorticoid-induced osteoporosis. N Engl J Med 2007;357:2028-39.

11. Hadji P, Zanchetta JR, Russo L, Recknor CP, Saag KG, McKiernan FE, et al. The effect of teriparatide compared with risedronate on reduction of back pain in postmenopausal women with osteoporotic vertebral fractures. Osteoporos Int 2012;23:2141-50.

12. Kendler DL, Marin F, Zerbini CAF, Russo LA, Greenspan SL, Zikan V, et al. Effects of teriparatide and risedronate on new fractures in post-menopausal women with severe osteoporosis (VERO): a multicentre, double-blind, double-dummy, randomised controlled trial. Lancet 2018;391:230-40.

13. Miller PD, Hattersley G, Riis BJ, Williams GC, Lau E, Russo LA, et al. Effect of abaloparatide vs placebo on new vertebral fractures in postmenopausal women with osteoporosis: a randomized clinical trial. JAMA 2016;316:722-33.

14. Langdahl BL, Libanati C, Crittenden DB, Bolognese MA, Brown JP, Daizadeh NS, et al. Romosozumab (sclerostin monoclonal antibody) versus teriparatide in postmenopausal women with osteoporosis transitioning from oral bisphosphonate therapy: a randomised, open-label, phase 3 trial. Lancet 2017;390:1585-94.

15. Bouxsein ML, Eastell R, Lui LY, Wu LA, de Papp AE, Grauer A, et al. Change in bone density and reduction in fracture risk: a meta-regression of published trials. J Bone Miner Res 2019;34:632-42.

16. Black DM, Bauer DC, Vittinghoff E, Lui LY, Grauer A, 
Marin F, et al. Treatment-related changes in bone mineral density as a surrogate biomarker for fracture risk reduction: meta-regression analyses of individual patient data from multiple randomised controlled trials. Lancet Diabetes Endocrinol 2020;8:672-82.

17. Cosman F, Nieves JW, Dempster DW. Treatment sequence matters: anabolic and antiresorptive therapy for osteoporosis. J Bone Miner Res 2017;32:198-202.

18. Cummings SR, Cosman F, Eastell R, Reid IR, Mehta M, Lewiecki EM. Goal-directed treatment of osteoporosis. J Bone Miner Res 2013;28:433-8.

19. Lewiecki EM, Cummings SR, Cosman F. Treat-to-target for osteoporosis: is now the time? J Clin Endocrinol Metab 2013;98:946-53.

20. Kanis JA, McCloskey E, Branco J, Brandi ML, Dennison E, Devogelaer JP, et al. Goal-directed treatment of osteoporosis in Europe. Osteoporos Int 2014;25:2533-43.

21. Miedany YE. Treat to target for osteoporosis: another step forward. Curr Rheumatol Rev 2014;10:99-105.

22. Cummings SR, Cosman F, Lewiecki EM, Schousboe JT, Bauer DC, Black DM, et al. Goal-directed treatment for osteoporosis: a progress report from the ASBMR-NOF working group on goal-directed treatment for osteoporosis. J Bone Miner Res 2017;32:3-10.

23. Lewiecki EM, Watts NB. Assessing response to osteoporosis therapy. Osteoporos Int. 2021 In Press.

24. The clinician's guide to prevention and treatment of osteoporosis. Osteoporos Int. 2021 In press.

25. Thomas T, Casado E, Geusens P, Lems WF, Timoshanko J, Taylor D, et al. Is a treat-to-target strategy in osteoporosis applicable in clinical practice? Consensus among a panel of European experts. Osteoporos Int 2020;31:2303-11.

26. Nogues X, Nolla JM, Casado E, Jodar E, Munoz-Torres M, Quesada-Gomez JM, et al. Spanish consensus on treat to target for osteoporosis. Osteoporos Int 2018;29:489-99.

27. Diez-Perez A, Adachi JD, Agnusdei D, Bilezikian JP, Compston JE, Cummings SR, et al. Treatment failure in osteoporosis. Osteoporos Int 2012;23:2769-74.

28. Wasnich RD, Miller PD. Antifracture efficacy of antiresorptive agents are related to changes in bone density. J Clin Endocrinol Metab 2000;85:231-6.

29. Cummings SR, Karpf DB, Harris F, Genant HK, Ensrud K, LaCroix AZ, et al. Improvement in spine bone density and reduction in risk of vertebral fractures during treatment with antiresorptive drugs. Am J Med 2002;112:281-9.

30. Hochberg MC, Greenspan S, Wasnich RD, Miller P, Thomp- son DE, Ross PD. Changes in bone density and turnover explain the reductions in incidence of nonvertebral fractures that occur during treatment with antiresorptive agents. J Clin Endocrinol Metab 2002;87:1586-92.

31. Lewiecki EM, Kendler DL, Davison KS, Hanley DA, Harris ST, McClung MR, et al. Western osteoporosis alliance clinical practice series: treat-to-target for osteoporosis. Am J Med 2019;132:e771-7.

32. Ferrari S, Libanati C, Lin CJF, Brown JP, Cosman F, Czerwinski E, et al. Relationship between bone mineral density T-score and nonvertebral fracture risk over 10 years of denosumab treatment. J Bone Miner Res 2019;34:1033-40.

33. Eastell R, Rosen CJ, Black DM, Cheung AM, Murad MH, Shoback D. Pharmacological management of osteoporosis in postmenopausal women: an Endocrine Society clinical practice guideline. J Clin Endocrinol Metab 2019;104:1595622.

34. Shoback D, Rosen CJ, Black DM, Cheung AM, Murad MH, Eastell R. Pharmacological management of osteoporosis in postmenopausal women: an Endocrine Society guideline update. J Clin Endocrinol Metab 2020;105:dgaa048.

35. Camacho PM, Petak SM, Binkley N, Diab DL, Eldeiry LS, Farooki A, et al. American Association of Clinical Endocrinologists/American College of Endocrinology clinical practice guidelines for the diagnosis and treatment of postmenopausal osteoporosis: 2020 update. Endocr Pract 2020;26 (Suppl 1):1-46.

36. Tsourdi E, Langdahl B, Cohen-Solal M, Aubry-Rozier B, Eriksen EF, Guanabens N, et al. Discontinuation of denosumab therapy for osteoporosis: a systematic review and position statement by ECTS. Bone 2017;105:11-7.

37. Lewiecki EM. Marcus and Feldman's osteoporosis. 5th ed. San Diego: Elsevier; 2021. Chapter 61, Evaluation of the osteoporosis patient; p. 1475-500.

38. Lewiecki EM, Laster AJ. Clinical review: clinical applications of vertebral fracture assessment by dual-energy X-ray absorptiometry. J Clin Endocrinol Metab 2006;91:4215-22.

39. Borges JLC, Sousa da Silva M, Ward RJ, Diemer KM, Yeap SS, Lewiecki EM. Repeating vertebral fracture assessment: 2019 ISCD official position. J Clin Densitom 2019;22:484-8.

40. Lewiecki EM, Binkley N, Bilezikian JP. Treated osteoporosis is still osteoporosis. J Bone Miner Res 2019;34:605-6.

41. Adler RA, El-Hajj Fuleihan G, Bauer DC, Camacho PM, Clarke BL, Clines GA, et al. Managing osteoporosis in patients on long-term bisphosphonate treatment: report of a task force of the American Society for Bone and Mineral 
Research. J Bone Miner Res 2016;31:16-35.

42. Cheung AM, McKenna MJ, van de Laarschot DM, Zillikens MC, Peck V, Srighanthan J, et al. Detection of atypical femur fractures. J Clin Densitom 2019;22:506-16.

43. Cummings SR, Black DM, Thompson DE, Applegate WB,
Barrett-Connor E, Musliner TA, et al. Effect of alendronate on risk of fracture in women with low bone density but without vertebral fractures: results from the Fracture Intervention Trial. JAMA 1998;280:2077-82. 